\title{
Tactical Service Selection with Runtime Aspects
}

\author{
Rene Ramacher and Lars Mönch \\ Chair of Enterprise-wide Software Systems \\ Univerity of Hagen, 58084 Hagen, Germany \\ \{Rene.Ramacher, Lars.Moench\}@FernUni-Hagen. de
}

\begin{abstract}
The quality of service (QoS) of a service composition is addressed by a QoS-aware service selection. In the presence of sophisticated service charging models a cost-minimized service selection can be obtained related to a number of requests expected for a service composition in a predefined planning horizon. A service selection that is used to execute requests throughout an entire planning horizon is called tactical. The majority of service selection models assume a deterministic service execution and therefore the need for runtime adaptions of a service composition to react on service failures or deviating QoS values is neglected. The challenge that is addressed with this paper is to develop a tactical service selection approach that anticipates runtime adaptions of a service composition. It is shown that the tactical service selection can be efficiently combined with an existing service reconfiguration method to achieve both runtime-related goals and tactical objectives.
\end{abstract}

Keywords: QoS-aware Service Selection, Uncertain QoS, Distributed Decision Making.

\section{Introduction and Related Work}

The QoS and the cost of a service composition are addressed by a QoS-aware service selection. In the literature related to service marketing sophisticated service charging models, including subscription-based charging, service bundling, and quantity discounts, are proposed. A QoS-aware service selection model for a cost-minimized service selection in the presence of sophisticated service charging models is presented in [5]. The cost-minimized service selection is related to an expected number of service invocations in a planning horizon to fairly compare a service charged on a subscription base and a service charged on a transaction base. The cost-minimization objective is a tactical objective because it is related to a set of service invocations that occur in the planning horizon. Accordingly, a service selection model that pursues tactical objectives is called tactical.

The tactical service selection model presented in [5] relies on the assumption of a deterministic service execution which is not realistic because in a real environment service failures occur and some of the QoS attributes are uncertain. Service reconfiguration approaches are proposed to deal with volatile and uncertain execution environments. Canfora et al. 3] propose the reconfiguration of a service composition to react on service failures and on QoS values that

S. Basu et al. (Eds.): ICSOC 2013, LNCS 8274, pp. 413 420, 2013.

(C) Springer-Verlag Berlin Heidelberg 2013 
deviate from their expected ones. Li et al. 4 propose a service reconfiguration approach that focuses on service failures. The efficiency of an online performed service reconfiguration is addressed. The approach of Canfora et al. is extended by Ramacher and Mönch in [6] to the reconfiguration of time-critical service compositions that suffer from uncertain response times. In contrast to a tactical service selection, a service reconfiguration is called an operative service selection because it is always related to the execution of a certain request. Because of this request-centric point of view, operative service selection models cannot account for tactical objectives as e.g. the cost minimization in the presence of sophisticated service charging models.

This paper proposes a tactical service selection that accounts for a volatile and uncertain environment by anticipating the operatively executed service reconfiguration. Using a general framework for distributed decision making systems [7], the tactical service selection aligns the service reconfiguration to the tactical objectives. This alignment is carried out in terms of restrictions concerning the services that are considered by the service reconfiguration. The generality of the proposed alignment allows the integration of any of the aforementioned service reconfiguration approaches within the proposed tactical service selection.

The remainder of this paper is organized as follows. Section 2 introduces the service selection model. The proposed tactical service selection approach is presented in Section 3 . Afterwards, a solution approach for the tactical service selection is developed in Section 4 . Section 5 briefly summarizes the experiments performed to evaluate the approach. Finally, Section 6 concludes the paper and provides future research topics.

\section{Service Selection Model}

The structure of a service composition is defined in terms of a process model that consists of abstract tasks $T=\left\{t_{1}, \ldots, t_{n}\right\}$. This paper considers only sequential process models in which the tasks $t_{1}, \ldots, t_{n}$ are executed successively. The execution starts by task $t_{1}$ and ends with $t_{n}$. The processing of a task $t_{i+1}$ can only be started after the execution of $t_{i}$ is completed. Extending the ideas of this paper to more general process models that also contain conditional branches and flow constructs is possible by adopting the concepts presented in 6 . 8 .

The functional requirements of a task have to be fulfilled by a concrete service that is used to execute the task. A service class $S_{i}$ consists of the services that fulfill the functional requirements of $t_{i}$. Hence, establishing the service classes corresponds to a service selection with respect to functional requirements. The service classes can be determined manually or by reasoning techniques that rely on semantically enriched service interfaces. The set of all services is denoted as $S=\bigcup_{t_{i} \in T} S_{i}$. A service binding $b$ determines the service $b\left(t_{i}\right) \in S_{i}$ that is used to execute the task $t_{i}$. Hence, the service binding $b$ is defined as the mapping:

$$
b: T \mapsto S, t_{i} \rightarrow b\left(t_{i}\right) \in S_{i} .
$$

In the remainder, we abbreviate the service $b\left(t_{i}\right)$ that is bound to $t_{i}$ with $b_{i}$. 
The cost of a service consists of its invocation-dependent transaction cost and its associated periodic fees. The transaction cost $c(s) \in \mathbb{R}^{+}$for a service $s \in S$ is charged each time $s$ is invoked. The periodic fee $S(s) \in \mathbb{R}^{+}$is an invocationindependent cost that has to be considered to use a service throughout a period. A service $s$ is charged transaction-based when $c(s)>0$ and $S(s)=0$ holds, while a subscription-based charging is applied for $s \in S$ in the case of $c(s)=0$ and $S(s)>0$. A reasonable service selection that deals with services that are charged on a transaction base and services that are charged on a subscription base needs to take into account the expected number of service invocations. The quantity $Q$ is the number of requests that are expected for the service composition within a predefined planning horizon.

The actual response time of a service $s \in S$ is $r(s) \in \mathbb{R}^{+}$. Since this time is only known after a service was executed, an expected response time $\tilde{r}(s) \in \mathbb{R}^{+}$ is used for an ex-ante performed service selection. In the presence of a globally constrained execution time $\bar{e}$, a service binding $b$ is determined such that the execution time restriction is met by taking into account the expected response times by:

$$
\sum_{t_{i} \in T} \tilde{r}\left(b_{i}\right) \leq \bar{e} .
$$

In (2), $\tilde{r}$ represents an expected response time that can be obtained e.g. by a quantil-based measure [6]. A service binding $b$ is called the primary service binding if $b$ is determined prior to the execution of a request. Because it is likely that the actual response time of a service will deviate from the estimated one, a service binding needs to be adjusted by a service reconfiguration during the execution of a request to avoid the violation of the end-to-end constrained execution time. The reconfiguration adjusts the currently applied service binding $b$ to take into account the actual execution process and the realization of the QoS attributes. An adjusted service binding is thus always related to a certain request and is therefore called operational. The operational service binding that is related to the request $\rho$ is denoted with $b^{\rho}$. Initially, the operational service binding is the primary service binding.

\section{Hierarchical Tactical Service Selection}

The integration of the tactical service selection and the operative service reconfiguration is carried out by utilizing the hierarchical architecture of distributed decision making (DDM) systems. According to Schneeweis [7], a two-stage DDM system is divided into a top- and a base-level. The coupling between the topand base-level is carried out by an instruction. The top-level determines an instruction that influences the base-level's decision. The top-level exploits an anticipation of the base-level to support the decision making. The anticipation is used to represent the characteristics of the base-level that are relevant to the top-level [7. Top-, base-, and the anticipated base-level are defined in terms of their action space, criterion, and information status. 
The tactical service selection (TSS) corresponds to the top-level while the service reconfiguration is represented by an operative service selection (OSS) that corresponds to the base-level. The TSS uses an instruction to align the OSS to the tactical objectives. An instruction is a vector of operational service classes $\widehat{S}_{i} \subseteq S_{i}$. The OSS selects the service $b_{i}^{\rho}$ from the operational service class $\widehat{S}_{i}$ that is used to execute the task $t_{i}$ during request $\rho$ is processed.

The decision making of the TSS is supported by an anticipation of the OSS. The anticipation captures the assumption of the TSS concerning the OSS's service selection for a task in a certain situation. The operational service classes are determined by the TSS such that the periodic cost and the cost expected for $Q$ invocations of the service composition are minimized. In the following, the action space, the criterion, and the information status of the top-, base-, and the anticipated base-level are introduced.

The action space of the TSS is defined as:

$$
A^{T}=P\left(S_{1}\right) \times \ldots \times P\left(S_{n}\right)
$$

where $P\left(S_{i}\right)$ denotes the power set of the service class $S_{i}$. According to the definition of the action space, an action $\widehat{S} \in A^{T}$ is represented by the vector $\widehat{S}=\left(\widehat{S}_{1}, \ldots, \widehat{S}_{n}\right)$ of operational service classes. The information status of the TSS at the decision time $t_{0}$ is $I_{t_{0}}^{T}=Q$ where $Q$ is the number of requests expected for the service composition.

The criterion of the TSS is divided into a private criterion $C^{T T}$ and a bottomup criterion $C^{T B}$ [7]. The bottom-up criterion represents the influence of the anticipated OSS on the decision making process of the TSS. The private criterion of the TSS is to minimize the periodic cost of the services that are included in the operational service classes. The periodic costs are determined as the sum of the periodic fees of the services selected by an action $\widehat{S}$, i.e.

$$
C^{T T}(\widehat{S})=\sum_{t_{i} \in T} \sum_{s_{i j} \in \widehat{S}_{i}} S\left(s_{i j}\right)
$$

The bottom-up criterion concerns the transaction costs of the service invocations to execute $Q$ requests of the service composition. Since the decision which service $s_{i j} \in \widehat{S}_{i}$ is actually invoked to execute the task $t_{i}$ is related to the OSS, an anticipated number of service invocations $\tilde{q}_{i j}$ is considered. Taking into account the anticipation $A F(I N)$ of the OSS with respect to the instruction $I N=\widehat{S}$, the bottom-up criterion is stated as:

$$
C^{T B}(A F(I N))=\sum_{t_{i} \in T} \sum_{s_{i j} \in \widehat{S}_{i}} c\left(s_{i j}\right) \tilde{q}_{i j}
$$

The action space of the OSS is defined as the set of mappings between the tasks $T$ and the operational service classes $\widehat{S}$ identified by the TSS:

$$
A^{B}=\operatorname{Map}(T, \widehat{S})=\left\{b \mid b: T \mapsto \bigcup_{i=1}^{n} \widehat{S}_{i}, t_{i} \rightarrow b\left(t_{i}\right) \in \widehat{S}_{i}, t_{i} \in T\right\} .
$$


The information status $I_{\Psi}^{B}=t_{\text {cur }}$ of the OSS captures the time $\Psi$ elapsed since the processing of a request has been started and the task $t_{c u r} \in T$ to be processed next.

The goal of the OSS is to obtain a service binding $b \in \operatorname{Map}(T, \widehat{S})$ that ensures a reliable execution of the service composition with respect to its end-to-end constrained execution time. Considering $\Psi$ and $t_{c u r}$, the service binding $b$ is determined such that the execution time restriction will be met, i.e.

$$
\Psi+\sum_{i=c u r}^{n} \tilde{r}\left(b_{i}\right) \leq \bar{e} .
$$

The OSS applies a cost-minimizing objective concerning the transaction costs. The criterion of the OSS is given by:

$$
C_{\Psi}^{B}(b)=\sum_{i=c u r}^{n} c\left(b_{i}\right) .
$$

The service selection of the OSS is anticipated by the TSS. The service selection $b_{i}^{\rho}$ of the OSS relies on the start time of the task $t_{i}$ during the execution of the request $\rho$. The actual start time of $t_{i}$ is unknown to the TSS because it depends on the actual response times of the services invoked to execute the tasks prior to $t_{i}$. Hence, a set of response time scenarios $\Omega$ is used to obtain a reasonable anticipation. A scenario $\omega \in \Omega$ represents a certain response time $r^{\omega}\left(s_{i j}\right)$ for each $s_{i j} \in S$. The response time $r^{\omega}\left(s_{i j}\right)$ is sampled from a response time distribution of the service $s_{i j}$.

The anticipated information status is given as $\tilde{I}_{t_{0}}^{B}=(\Omega, Q)$. The goal of the anticipated OSS is to determine a service binding for each $\omega \in \Omega$ such that the execution time restriction of the service composition is met. Hence, the action space of the anticipated OSS is $\operatorname{Map}(T, \widehat{S})^{|\Omega|}$. Let $b \in \operatorname{Map}(T, \widehat{S})^{|\Omega|}$ be an action of the anticipated OSS. Then $b^{\omega}: T \rightarrow \bigcup_{i=1}^{n} \widehat{S}_{i}$ is the operational service binding for scenario $\omega$ and $b_{i}^{\omega} \in \widehat{S}_{i}$ is the service used to execute the task $t_{i}$ in $\omega$.

The anticipated criterion of the OSS considers the transaction cost resulting from the execution of all scenarios. For an action $b \in \operatorname{Map}(T, \widehat{S})^{|\Omega|}$, the criterion is stated as:

$$
\tilde{C}^{B}(b)=\sum_{\omega \in \Omega} \sum_{t_{i} \in T} c\left(b_{i}^{\omega}\right) .
$$

Moreover, the execution time restrictions for the scenarios in $\Omega$ are state as:

$$
\sum_{t_{i} \in T} r^{\omega}\left(b_{i}^{\omega}\right) \leq \bar{e}, \omega \in \Omega
$$

The anticipated number of invocations $\tilde{q}_{i j}$ of the service $s_{i j}$ is derived from the decision of the anticipated OSS as:

$$
\tilde{q}_{i j}=\frac{Q}{|\Omega|} \cdot \sum_{\omega \in \Omega} e_{i \omega}\left(s_{i j}\right)
$$

where $e_{i \omega}\left(s_{i j}\right)=1$ when $b_{i}^{\omega}=s_{i j}$ and otherwise 0 . 


\section{Integrated Solution Approach}

This section presents a solution approach used to obtain the operational service classes such that the objectives of the TSS, stated by (4) and (5), are achieved. A possible implementation of the OSS is described in [1]618.

The scenarios used to capture the uncertainty of the response times are linked together by nonanticipativity constraints [2]. The nonanticipativity constraints ensure that the same decisions are taken in all scenarios that are indistinguishable at the decision time. Two scenarios $\omega$ and $\omega^{\prime}$ are indistinguishable in the case of an operational service selection if the difference of the start time of $t_{i}$ in $\omega$ and $\omega^{\prime}$ is less than a threshold $\tau$ used to initiate a service reconfiguration. In this situation, the same service has to be selected for task $t_{i}$ in $\omega$ and $\omega^{\prime}$.

The TSS is implemented as the mixed integer program (MIP) formulated through (12)-(21). In the MIP, the service selection is captured by the binary decision variables $e_{i j}$ and $z_{i j}^{\omega}$. The variables $e_{i j}$ represent the decision of the TSS. The value of $e_{i j}$ is 1 if the service $s_{i j} \in S_{i}$ is included in the operational service class $\widehat{S}_{i}$, otherwise it is 0 . The operational service selection in scenario $\omega \in \Omega$ is captured by the variables $z_{i j}^{\omega}$. The variable $z_{i j}^{\omega}$ is set to 1 if the service $s_{i j}$ is used to execute the task $t_{i}$ in the scenario $\omega$, otherwise $z_{i j}^{\omega}$ is set to 0 . The real-valued decision variables $\tilde{q}_{i j}$ represent the expected number of invocations of the service $s_{i j}$ according to (11). The start time of a task $t_{i}$ in a scenario $\omega$ is modeled by the real-valued decision variable $a_{i}^{\omega}$. The binary decision variables $\sigma_{i}^{\omega, \omega^{\prime}}$ are used to implement the nonanticipativity constraints. The value of $\sigma_{i}^{\omega, \omega^{\prime}}$ is 1 if the difference of the start time of $t_{i}$ in the scenarios $\omega$ and $\omega^{\prime}$ is larger than the threshold $\tau$, otherwise it is 0 . We obtain:

$$
\min \sum_{t_{i} \in T} \sum_{s_{i j} \in S_{i}} S\left(s_{i j}\right) e_{i j}+\sum_{t_{i} \in T} \sum_{s_{i j} \in S_{i}} c\left(s_{i j}\right) \tilde{q}_{i j}
$$

subject to:

$$
\begin{gathered}
\sum_{s_{i j} \in S_{i}} z_{i j}^{\omega}=1, \forall \omega \in \Omega, t_{i} \in T \\
a_{i}^{\omega} \geq 0, \forall \omega \in \Omega, t_{i} \in T \\
a_{i+1}^{\omega} \geq a_{i}^{\omega}+\sum_{s_{i j} \in S_{i}} r^{\omega}\left(s_{i j}\right) z_{i j}^{\omega}, \forall \omega \in \Omega, t_{i} \in T \backslash\left\{t_{n}\right\} \\
\sum_{t_{i} \in T} \sum_{s_{i j} \in S_{i}} r^{\omega}\left(s_{i j}\right) z_{i j}^{\omega} \leq \bar{e}, \forall \omega \in \Omega \\
z_{i j}^{\omega} \leq e_{i j}, \forall \omega \in \Omega, i \in T, s_{i j} \in S_{i} \\
\tilde{q}_{i j}=\frac{Q}{|\Omega|} \sum_{\omega \in \Omega} z_{i j}^{\omega}, \forall t_{i} \in T, s_{i j} \in S_{i} \\
a_{i}^{\omega}-a_{i}^{\omega^{\prime}}-\tau \geq M\left(\sigma_{i}^{\omega \omega^{\prime}}-1\right), \forall \omega, \omega^{\prime} \in \Omega, i \in T
\end{gathered}
$$




$$
\begin{gathered}
z_{i j}^{\omega}-z_{i j}^{\omega^{\prime}} \leq \sigma_{i}^{\omega, \omega^{\prime}}+\sigma_{i}^{\omega^{\prime}, \omega}, \forall \omega, \omega^{\prime} \in \Omega, t_{i} \in T, s_{i j} \in S_{i} \\
z_{i j}^{\omega}, e_{i j} \in\{0,1\}, \forall \omega \in \Omega, t_{i} \in T, s_{i j} \in S_{i} .
\end{gathered}
$$

The objective function (12) concerns the total cost resulting from the periodic fees of the services that are included in the operational service classes and the transaction costs incurred by $Q$ service invocations according to (4) and (5).

The constraints (13)-(16) account for the operational service selection. First, the equations (13) ensure that exactly one service is selected for each task in each scenario. According to the inequalities (14), the start time of each task has to be non-negative. In addition, the start time of a task $t_{i+1}$ is determined by the completion time of its preceding task $t_{i}$ which is ensured by the inequalities (15). In (15), the completion time of the preceding task $t_{i}$ is calculated as the sum of its start time and the scenario-related response time of the service that is selected to execute $t_{i}$. The inequalities (16) account for the execution time restriction that has to be fulfilled for each scenario $\omega \in \Omega$ according to (2).

The inequalities (17) and (18) are the constraints to couple the TSS with the action space of the anticipated OSS. According to (17), the service $s_{i j}$ can be selected to execute task $t_{i}$ only if $s_{i j}$ is element of the operational service class $\widehat{S}_{i}$. The equations (18) are used to obtain the estimated number of invocations $\tilde{q}_{i j}$ of each service $s_{i j}$.

The inequalities (19) and (20) are the nonanticipativity constraints. According to (19), the value of $\sigma_{i}^{\omega, \omega^{\prime}}$ is 1 only if the difference of the start time of task $t_{i}$ in the scenarios $\omega$ and $\omega^{\prime}$ exceeds the threshold $\tau$. If this is the case then the left-hand side will be positive and $\sigma_{i}^{\omega, \omega^{\prime}}$ is set to 1 . Otherwise, the value of $\sigma_{i}^{\omega, \omega^{\prime}}$ has to be 0 . With respect to (20) a different service can be selected in $\omega$ and $\omega^{\prime}$ for $t_{i}$ only if either $\sigma_{i}^{\omega, \omega^{\prime}}$ or $\sigma_{i}^{\omega^{\prime}, \omega}$ takes a value of 1 . Otherwise, the right-hand side of (20) is 0 forcing that the same service is selected for $t_{i}$ in $\omega$ and $\omega^{\prime}$.

\section{Computational Experiments}

Experiments are conducted to evaluate the TSS model with respect to its computational tractability and the anticipation of the operational service selection. The experiments are performed on randomly generated problem instances. A detailed description of the experiments is not included due to space restrictions. The experiments show that a tactical service selection can be obtained for service compositions containing up to 20 tasks in less than one hour of computing time. An anticipation with 30 scenarios allows a successful execution for almost all requests. In contrast, the number of successfully executed requests decreases dramatically when no anticipation is used by the TSS. The quality of the anticipation with respect to the transaction cost increases with an increasing number of scenarios. However, the number of scenarios is restricted by an increasing computational burden that is required to solve the TSS model. 


\section{Conclusion}

A tactical service selection is presented that addresses a cost-minimized service selection in the presence of sophisticated service charging models. Exploiting the concepts of DDM systems, the proposed tactical service selection integrates an operatively performed service reconfiguration by anticipation to consider uncertain response times and their impact on an end-to-end constrained execution time. The service reconfiguration is aligned to the tactical objectives and a successful service execution is ensured. In future research, an approach based on a metaheuristic will be developed to decrease the computational burden required to solve the TSS model for large scale service compositions.

\section{References}

1. Ardagna, D., Pernici, B.: Adaptive service composition in flexible processes. IEEE Trans. Software Engineering 33(6), 369-384 (2007)

2. Birge, J.R., Louveaux, F.: Introduction to Stochastic Programming. Series in Operations Research and Financial Engineering. Springer (1997)

3. Canfora, G., Di Penta, M., Esposito, R., Villani, M.L.: QoS-aware replanning of composite web services. In: Proceedings of the IEEE International Conference on Web Services, pp. 121-129 (2005)

4. Li, J., Ma, D., Mei, X., Sun, H., Zheng, Z.: Adaptive QoS-aware service process reconfiguration. In: Proceedings of the 8th International Conference on Services Computing (SCC), pp. 282-289. IEEE Computer Society, Washington, DC (2011)

5. Ramacher, R., Mönch, L.: Cost-minimizing service selection in the presence of endto-end QoS constraints and complex charging models. In: Proceedings of the 9th International Conference on Services Computing (SCC), pp. 154-161 (2012)

6. Ramacher, R., Mönch, L.: Reliable service reconfiguration for time-critical service compositions. In: Proceedings of the 10th International Conference on Services Computing (SCC), pp. 184-191 (2013)

7. Schneeweiss, C.: Distributed Decision Making. Springer (2003)

8. Yu, T., Zhang, Y., Lin, K.-J.: Efficient algorithms for web services selection with end-to-end QoS constraints. ACM Transactions on the Web (TWeb) 1(1) (2007) 